\title{
Influence of the Parameterization in the Interval Solution of Elastic Beams
}

\author{
Stefano Gabriele and Valerio Varano \\ Modelling and Simulation LAB (Lams), Roma Tre University, Via della Madonna dei Monti 40, 00184 Rome, Italy \\ Correspondence should be addressed to Stefano Gabriele; stefano.gabriele@uniroma3.it
}

Received 29 May 2014; Accepted 15 September 2014; Published 24 September 2014

Academic Editor: Greg Foliente

Copyright (C) 2014 S. Gabriele and V. Varano. This is an open access article distributed under the Creative Commons Attribution License, which permits unrestricted use, distribution, and reproduction in any medium, provided the original work is properly cited.

We are going to analyze the interval solution of an elastic beam under uncertain boundary conditions. Boundary conditions are defined as rotational springs presenting interval stiffness. Developments occur according to the interval analysis theory, which is affected, at the same time, by the overestimation of interval limits (also known as overbounding, because of the propagation of the uncertainty in the model). We suggest a method which aims to reduce such an overestimation in the uncertain solution. This method consists in a reparameterization of the closed form Euler-Bernoulli solution and set intersection.

\section{Introduction}

While dealing with real problem solutions, during some structural engineering procedures, uncertainties must be taken into consideration.

One example is the presence of epistemic uncertainties due to lack of knowledge for what concerns physical phenomena under study. This is an issue which could be included among the problem equations under the label of uncertain parameters.

At this stage it is necessary to introduce a representation of such parameters according to a theory of uncertainty.

The classical way of proceeding is to represent aleatory uncertainties as stochastic variables and solving the problem in two different ways: through a probabilistic analysis if equations are allowed to be integrated or through a Monte Carlo approach, if a numerical solution is required.

Nowadays the increasing tendency is to represent epistemic uncertainty through means of nonprobabilistic methods with an uncertain interval analysis [1].

In particular interval analysis [2] is fascinating because it gives the possibility to compute uncertain bounded solutions with the certainty, under defined conditions, to include all the possible solutions which can be obtained from our model. All the practical implications of such property can be found both in the formulation of interval global optimization algorithms [3] and in the solution of inverse engineering problems [4].
It is commonly known that the main drawback of interval analysis is the so-called dependency effect, which leads to a deep overestimation (also called overbounding) of the interval solution bounds.

This could represent a serious problem since, even though all possible solutions are included with certainty, very large bounds generally make the result meaningless from a physical point of view, and hence useless from an engineering point of view. This is the reason why there is a diffused attempt, together with the community of people who deal with interval analysis based applications, to reduce such overbounding [5]. Very recently a new method has been presented by Elishakoff and Miglis [6]. This method, based on a trigonometric parameterization of the intervals, does not lead to an overbounded solution of truss structures.

In this paper we propose a method in order to reduce the interval overestimation, based on a varied parameterization of functions' domain (that is reparameterization) and interval set intersections. Our discussion about this method is based on its application to a classical benchmark, that is, the simple solution of an Euler-Bernoulli elastic beam with uncertain boundary conditions, by providing theoretical developments and numerical example. The proposed procedure is compared with a standard interval solution and well-known interval approach, called interval hull method which has been used to decrease the overestimation. Computational issues will be discussed, as well. 


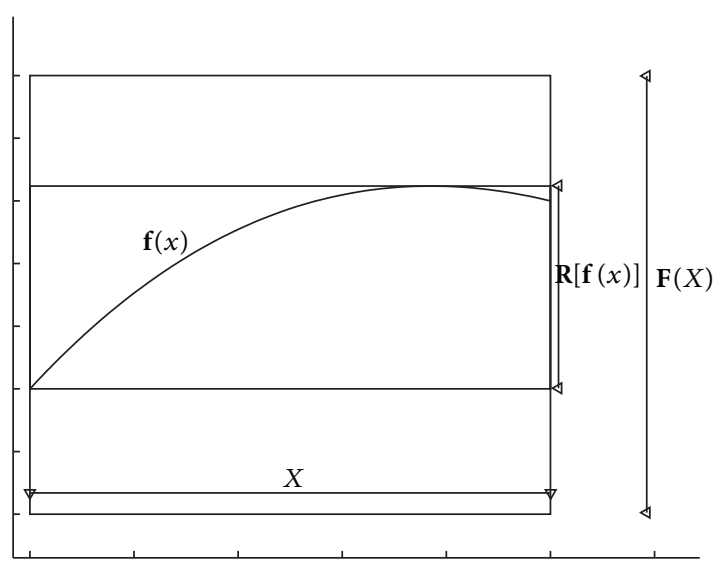

(a)

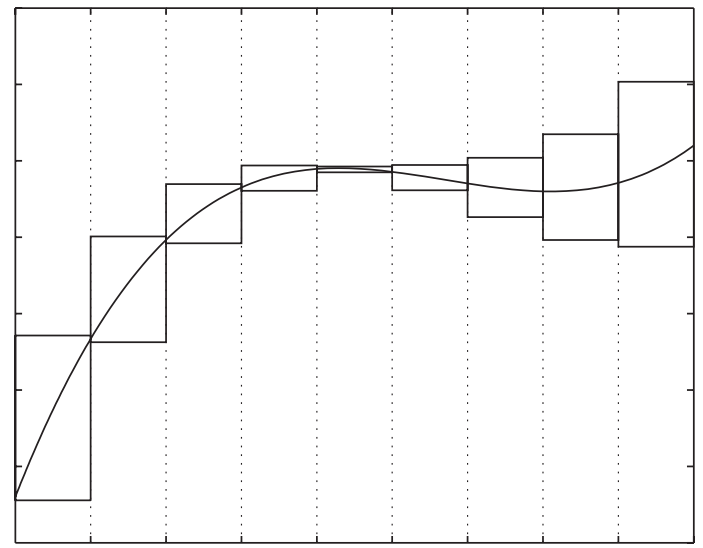

(b)

FIGURE 1: (a) Different overbounding of interval function. (b) Overbounding reduction by interval hull method (HM).

\section{Interval Analysis}

One key point is the definition of interval functions, called interval extensions. As Moore affirms in his book [2], interval extensions are not unique, and different interval extensions of the same function lead to different interval solution bounds with different overestimations. The overbounding cannot be eliminated but can be reduced during the practical phase.

In the following we denote, in bold letters, any uncertain quantity as an interval $\mathbf{X}=\left[x_{\text {inf }}, x_{\text {sup }}\right]=x_{c}+\Delta x[-1,1]$, inf and sup representing the interval limits, $x_{c}$ being the interval midpoint, and $\Delta x$ being the associated uncertainty, that is, the interval radius.

The main purpose of the evaluation of interval functions is to estimate the function range $\mathbf{R}=[\min f(x), \max f(x)]_{\mathbf{X}}$, without solving $\min$ and $\max$ problems. This is possible through interval analysis, with the previously mentioned drawback of the overestimation of the function range (conservative approach). Interval function extensions can be defined with awareness.

Considering a real valued function $f(x)$ such that $f$ : $\mathbb{R}_{n} \rightarrow \mathbb{R}$ we call interval extension the function $\mathbf{f}(\mathbf{x})$ the mapping $\mathbf{f}: \mathbb{\mathbb { R } _ { n }} \rightarrow \mathbb{R}$, such as $\mathbf{f}\left(x_{c}\right)=f\left(x_{c}\right), \mathbb{R}$ being the real interval space.

Interval extensions are not unique. For example, for every natural extension a real uncertain variable is replaced by its interval counterpart; centered forms and mean value forms are, respectively, interval extensions based on midpoint value and first order derivative evaluation [2]. Different kind of interval extensions can be defined to obtain a different level of refinement in the sought solution, namely, an interval in the function codomain. It is out of the purposes of this paper to compare the solution among these different choices. For this reason we choose the straightforward way of the "natural extension" that maintains the structure of the original equations and allows discussing the mechanical relevance of the uncertain quantities.

In general differences in the functional form of natural extensions lead to differences in the uncertainty propagation, that finally affect the uncertainty associated with the estimation of the function range $\mathbf{R}$, that might be largely overestimated. From an engineering point of view interval functions represent physical-mathematical model equations. The larger the evaluated function uncertainty is, due to the overestimation, the poorer the interpretation of the result could be.

For example we can consider the functions representation in Figure 1. Figure 1(a) shows two interval extensions $\mathbf{F}(x)$ and $\mathbf{R}[\mathbf{f}(x)]$ evaluated on the same interval $\mathbf{X}$. Both extensions lead to a complete inclusion of the whole function range, but only the second one coincides with it.

On the other hand, Figure 1(b) represents the application of one of the main methods used to reduce interval overbounding: the interval hull method (HM) [7]. Supposing that $\mathbf{x}$ is subdivided into disjoined subintervals $\mathbf{x}_{i}$ such that,

$$
\mathbf{x}=\bigcup_{i} \mathbf{x}_{i}
$$

then the interval hull $\mathbf{Y}$ includes the range $\mathbf{R}$ and can be defined as

$$
\mathbf{Y}=\bigcup_{i} \mathbf{f}\left(\mathbf{x}_{i}\right) \supseteq \mathbf{R}[f(x)]_{X}
$$

From now on the hull method will be used as the main solution for any other confrontation with the proposed method.

\section{Numerical Example}

In this section, we discuss the use of interval analysis in solving the simple structural problem of an elastic beam with uncertain boundary conditions, represented by elastic springs endowed with interval stiffness. In many applications structural joints are very different with respect to the way that they are modeled (e.g., elastic springs) and joints parameter identification is a key problem in the modeling of complex structures [8].

The uncertain quantities are inserted in the problem equations as interval variables and the provided solution is 


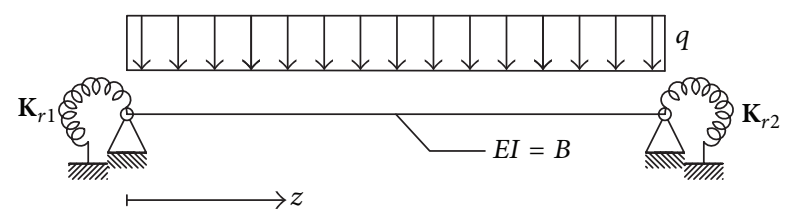

FIGURE 2: The selected structural problem.

discussed according to the theory of interval analysis [2] The expected uncertain solution is represented by the interval bound of the displacement field $\mathbf{v}(z)$.

The problem equations follow from the static solution of a supported beam with rotational springs at ends (Figure 2), according to the Euler-Bernoulli beam theory.

The uncertain spring parameters $\mathbf{K}_{r 1}$ and $\mathbf{K}_{r 2}$ act on the boundary conditions as follows:

$$
\begin{array}{cc}
v(0)=0, & v(L)=0, \\
\phi(0)=-\frac{\chi(0) B}{\mathbf{K}_{r 1}}, & \phi(L)=\frac{\chi(L) B}{\mathbf{K}_{r 2}} .
\end{array}
$$

$\chi(z)$ is the beam curvature and $B$ the elastic flexural stiffness.

Here we present the standard interval solution that is found by using the interval natural extension of the closed form solution of the problem in Figure 2. In the selected case the solution is given by a fourth order polynomial:

$$
\mathbf{v}(z)=\mathbf{C}_{0}+\mathbf{C}_{1} z+\mathbf{C}_{2} z^{2}+\mathbf{C}_{3} z^{3}+C_{4} z^{4}
$$

with $\left\{\mathbf{C}_{0}, \mathbf{C}_{1}, \mathbf{C}_{2}, \mathbf{C}_{3}\right\}$ being interval coefficients that depend on the uncertain parameters $\mathbf{K}_{r 1}$ and $\mathbf{K}_{r 2}$. An example is given in (5), where the analytical expression of $\mathbf{C}_{3}$ is given:

$$
\mathbf{C}_{3}=\frac{q L^{2}}{12} \frac{\left(\mathbf{K}_{r 2}-\mathbf{K}_{r 1}\right)}{12 B^{2}-4 B L\left(\mathbf{K}_{r 1}+\mathbf{K}_{r 2}\right)+\mathbf{K}_{r 1} \mathbf{K}_{r 2} L^{2}} .
$$

Figure 3 depicts the interval solution obtained from (4), when $B=1000 \mathrm{kNm}^{2}, q=10 \mathrm{kN} / \mathrm{m}, \mathbf{K}_{r 1}=[0.9,1.1] \mathrm{kNm}$, and $\mathbf{K}_{r 2}=[0.9,1.1] \times 10^{5} \mathrm{kNm}$, by applying directly the interval analysis rules given in Moore et al. [2] for the evaluation of interval polynomials.

It is evident in Figure 3 the large overestimation of the displacement field and how the overbounding increases towards the right side. In particular looking at the right boundary condition it should physically be from (3) $v(L)=0$. The value $v(L)=0$ is included in the interval solution but not limited to when $z / L=1$. This shows that the natural interval solution includes both physical and nonphysical solutions.

\section{Effect of Reparameterization}

The correction of the solution given in the previous section is here proposed through a reparameterization of (4) that is obtained by defining a new abscissa $s=z+z_{0}, z_{0}$ being the origin location of the beam local axis. It must be stressed that this does not correspond to a new representation of the

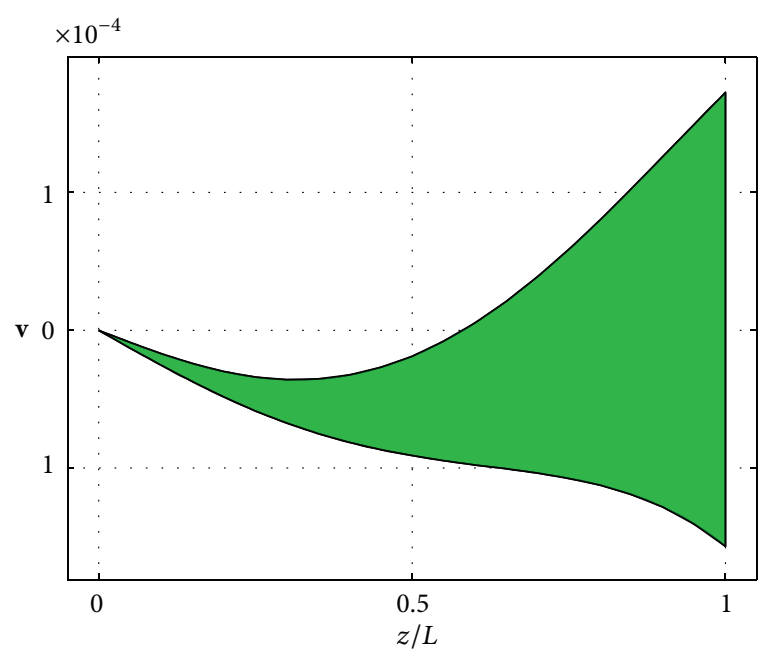

FIGURE 3: Interval solution of the displacement field $\mathbf{v}$.

interval quantities, but it is simply a reparameterization of the function's domain. This is important because all interval properties remain unchanged from this reparameterization, the inclusion property in particular, which provides a guarantee for the inclusion of all the possible solutions.

From this position (6) follows, where the interval coefficients dependence from $z_{0}$ is made explicit, and (7) reports the new analytical expression of the coefficient $\mathrm{C}_{3}$, which coincides with the expression in (5) as $z_{0}=0$ :

$$
\begin{aligned}
& \mathbf{v}\left(s, z_{0}\right)= \mathbf{C}_{0}\left(\mathbf{K}_{r i}, z_{0}\right)+\mathbf{C}_{1}\left(\mathbf{K}_{r i}, z_{0}\right) s \\
&+\mathbf{C}_{2}\left(\mathbf{K}_{r i}, z_{0}\right) s^{2}+\mathbf{C}_{3}\left(\mathbf{K}_{r i}, z_{0}\right) s^{3}+C_{4} s^{4} \\
& \mathbf{C}_{3}=\frac{q L^{2}}{12}\left(\frac{(6)}{12 B^{2}-4 B L\left(\mathbf{K}_{r 1}+\mathbf{K}_{r 2}\right)+\mathbf{K}_{r 1} \mathbf{K}_{r 2} L^{2}}-\frac{2 z_{0}}{B L^{2}}\right) .
\end{aligned}
$$

From the interval analysis point of view, interval functions defined by (6) can be considered as a family of different extensions of $\mathbf{v}$ depending on the choice of the parameter $z_{0}$.

Due to the nonuniqueness of the interval extension, different choices of $z_{0}$ will lead to different interval solution. This is shown in Figure 4, where three different displacement fields are obtained, respectively, for $z_{0}=0, L / 4, L / 2$.

It can be noticed, from Figure 4 , that each parameterization of the function will lead to a solution that has a different overbounding degree, depending on the function branch analyzed in that moment. Moreover, since an interval solution can be considered as a particular uncertainty set, the set intersection of the obtained solutions can be considered again a result which has all the properties of a general interval solution, the inclusion property in particular. In other words if every solution, obtained for different values of $z_{0}$, is able to include all the possible values of the range of $\mathbf{v}(z)$, then the set intersection of all the solutions will again include such a range.

Denoting as $\mathbf{R}[v]$ the range of the displacement field $\mathbf{v}(z)$, and $\mathbf{v}\left(s, z_{0 i}\right)$ being the displacement field obtained from the 


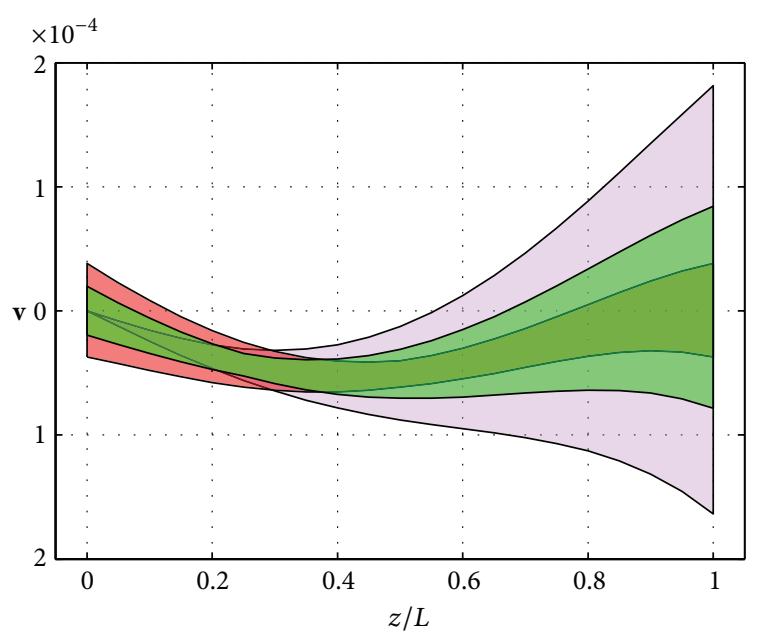

Figure 4: Different displacement fields obtained for $z_{0}=0, L / 4$, $L / 2$.

$i$ th choice of $z_{0}=\left\{z_{01}, \ldots, z_{0 i}, \ldots, z_{0 n}\right\}$, then the interval solution of the displacement field can be calculated as follows:

$$
\mathbf{v}(z)=\bigcap_{i} \mathbf{v}\left(s, z_{0 i}\right) \supseteq \mathbf{R}[v] .
$$

Equation (8) exploits the same principle of (2), by using the set intersection instead of a set union. Solution obtained from (8) is also called reparameterization interval (RPI). The RPI solution is finally compared to the one given by the hull method of (2), which is usually used to reduce interval overbounding.

Figure 5 shows the comparison of the two methods. An equal number of samples $(n=20)$ has been chosen to make the comparison meaningful, $n$ being the number of different $z_{0 i}$ choices or the number of evaluated $\mathbf{K}_{r i}$ subintervals, in case of RPI or HM, respectively.

From Figure 5 follows that HM is able to give sharper bounds with the same number of samples, but RPI solution is better from a qualitative point of view since boundary conditions are satisfied. The situation changes for what concerns the HM solution at right side boundary. In any case the overbounding reduction obtained from RPI is significant as well.

\section{Conclusions}

A new interval method aimed at reducing interval overbounding has been presented and exemplified through the application to a specific class of structural problems. In particular it is applied to find the sharp uncertain displacement field of beams with uncertain boundary conditions. The method (RPI) is based on the domain reparameterization of the analytical expression of the solution and is able to significantly reduce the overbounding by preserving some physical assumption such as fixity in different boundary conditions. The effectiveness of the presented method is showed by comparison with a classical literature method (HM).
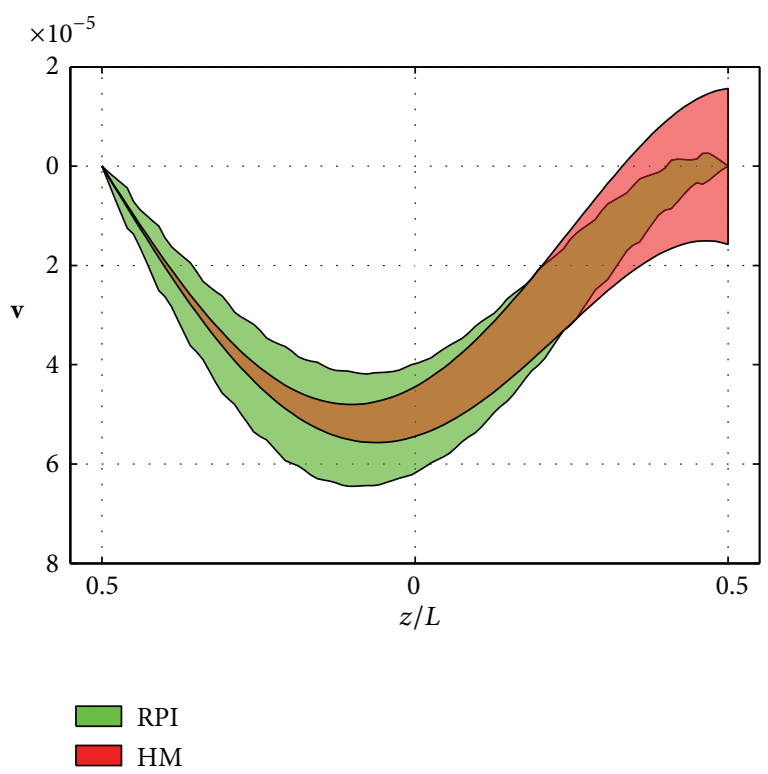

FigURE 5: RVI solution versus HM solution $(n=20)$.

The method is here presented for the cases when a closed form solution of the problem is known. This limitation does not put in jeopardy the qualitative results, which could be further generalized for the application to finite element methods, acting on the element shape functions whose expressions are known. Further developments will be hence devoted to generalize the Interval Finite Element method for beam structures and will allow for applications to every kind of framed structures, where boundary conditions (external or between elements) are uncertain.

\section{Conflict of Interests}

The authors declare that there is no conflict of interests regarding the publication of this paper.

\section{References}

[1] L. P. Swiler, T. L. Paez, and R. L. Mayes, "Epistemic uncertainty quantification tutorial," in Proceedings of the 27th Conference and Exposition on Structural Dynamics ( IMAC '09), Society for Experimental Mechanics, Orlando, Fla, USA, February 2009.

[2] R. E. Moore, R. B. Kearfott, and M. J. Cloud, Introduction to Interval Analysis, SIAM Press, Philadelphia, Pa, USA, 2009.

[3] E. Hansen and G. W. Walster, Global Optimization Using Interval Analysis, vol. 264 of Monographs and Textbooks in Pure and Applied Mathematics, Marcel Dekker, New York, NY, USA, 2004.

[4] S. Gabriele and C. Valente, "An interval-based technique for FE model updating," International Journal of Reliability and Safety, vol. 3, no. 1-3, pp. 79-103, 2009.

[5] E. Hansen, "Sharpening interval computations," Reliable Computing, vol. 12, no. 1, pp. 21-34, 2006.

[6] I. Elishakoff and Y. Miglis, "Novel parameterized intervals may lead to sharp bounds," Mechanics Research Communications, vol. 44, pp. 1-8, 2012. 
[7] S. Gabriele and A. Culla, "Comparison of statistical and interval analysis for the energy flow uncertainties in structural vibrating systems," Journal of Sound and Vibration, vol. 314, no. 3-5, pp. 672-692, 2008.

[8] Q. Guo and L. Zhang, "Identification of the mechanical joint parameters with model uncertainty," Chinese Journal of Aeronautics, vol. 18, no. 1, pp. 47-52, 2005. 

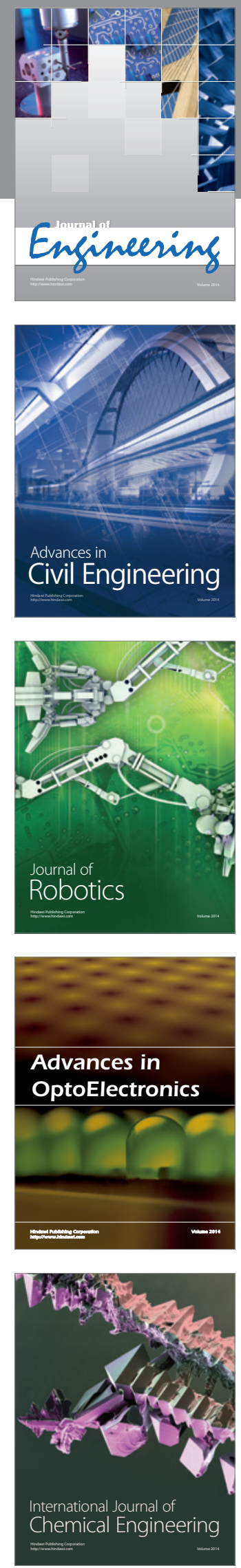

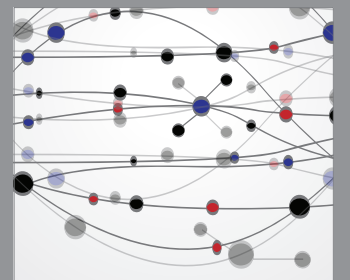

The Scientific World Journal
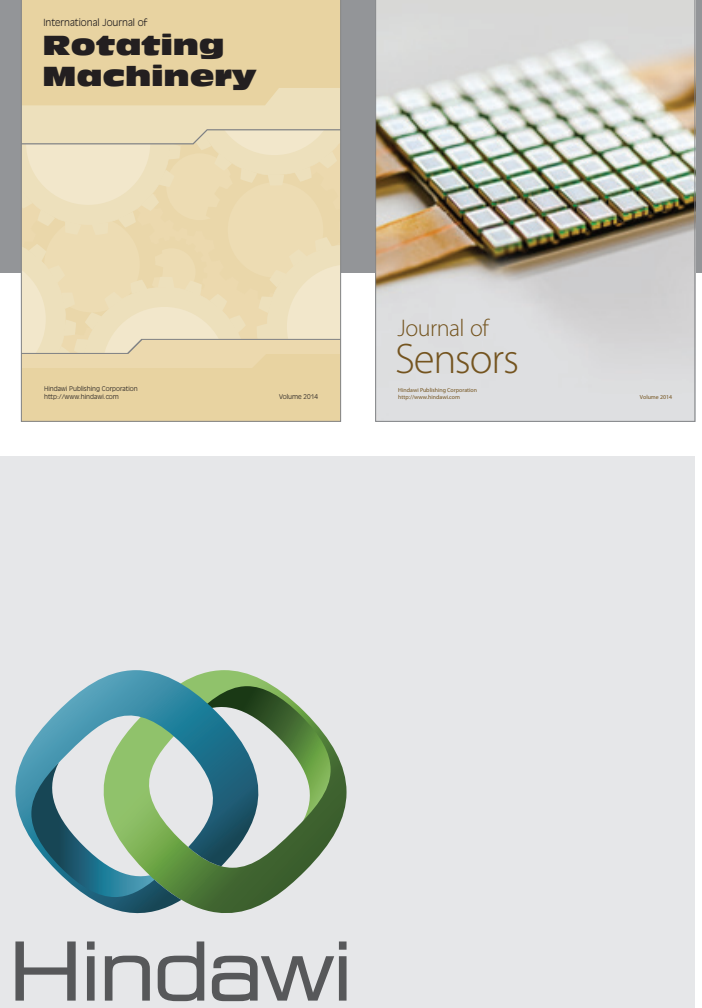

Submit your manuscripts at http://www.hindawi.com
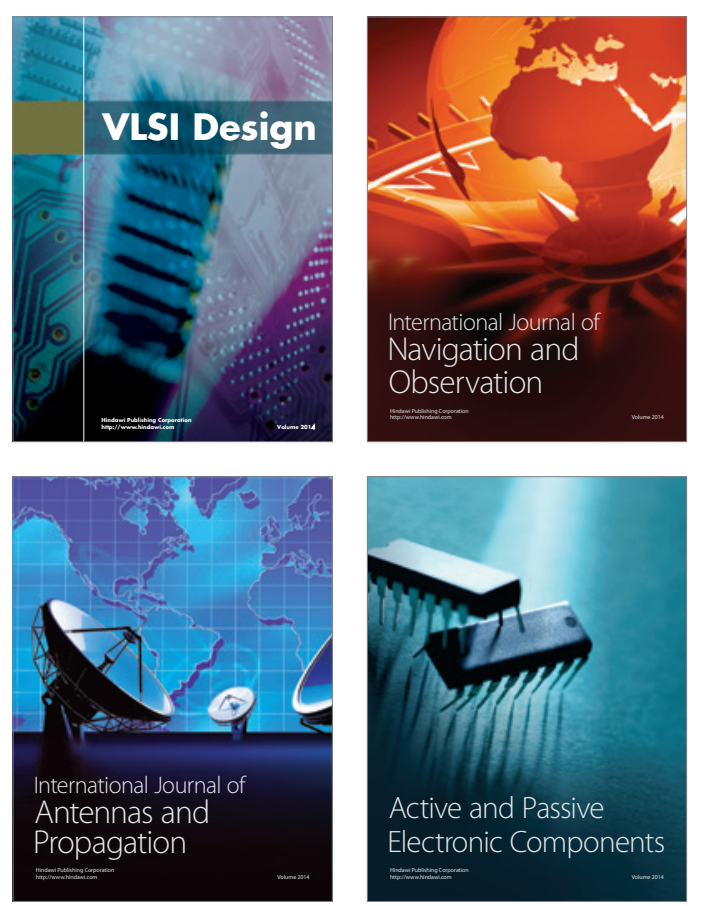
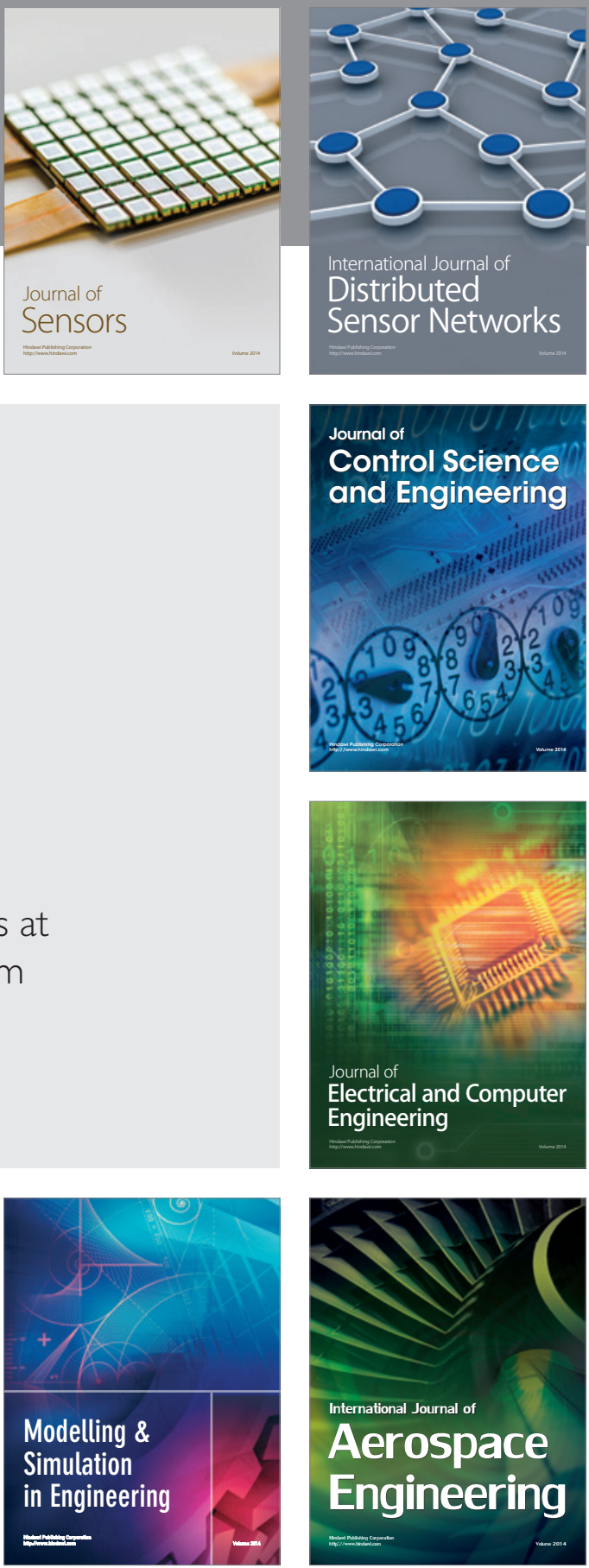

Journal of

Control Science

and Engineering
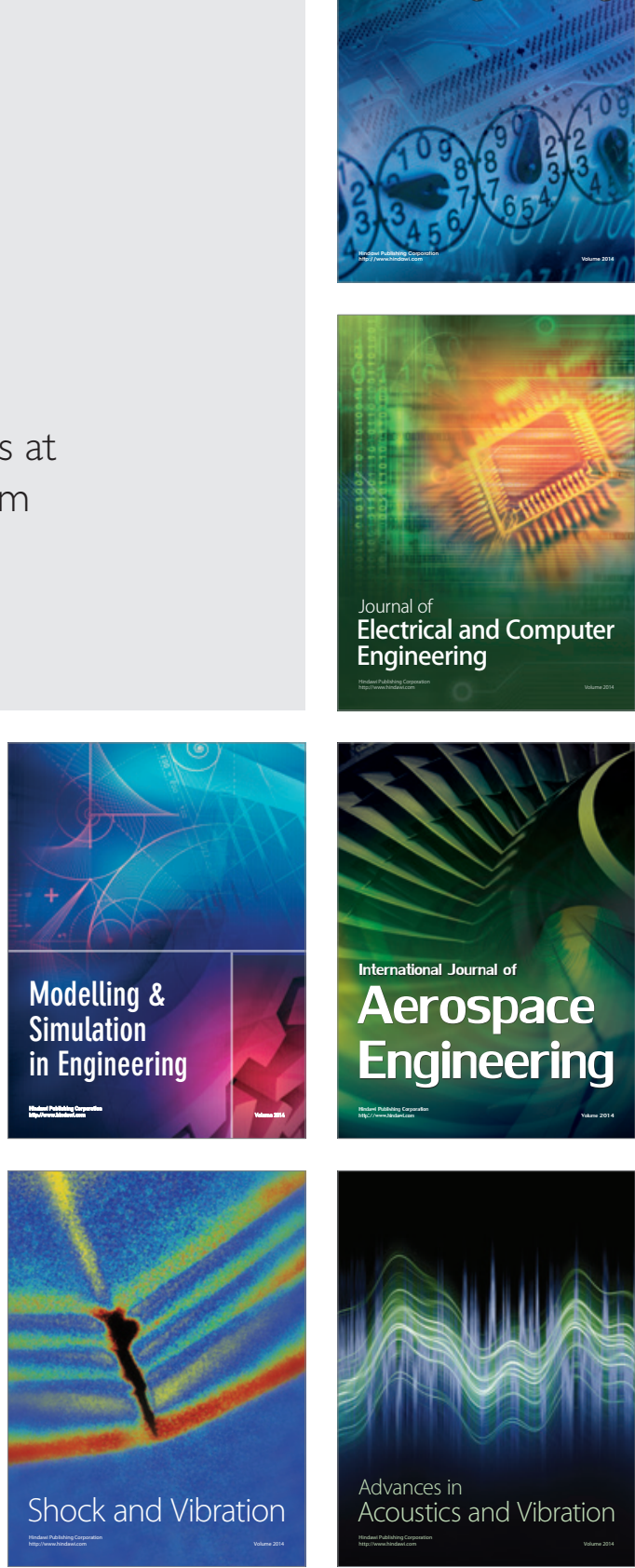